\title{
ORIENTAÇÃO E ORTORRETIFICAÇÃO DE IMAGENS TERRESTRES NADIRAIS
}

\section{Orientation and orthorectification of nadiral terrestrial images}

\author{
ADILSON BERVEGLIERI \\ MARCUS VINÍCIUS ANTUNES DE MORAES \\ ANTONIO MARIA GARCIA TOMMASELLI
}

Programa de Pós-Graduação em Ciências Cartográficas

Faculdade de Ciências e Tecnologia - Departamento de Cartografia

UNESP - Univ Estadual Paulista

Rua Roberto Simonsen, 305, CEP 19060-900 Presidente Prudente, SP

\{adilsonberveg, antunesdemoraes\}@ gmail.com, tomaseli@ fct.unesp.br

\begin{abstract}
RESUMO
Informações de controle terrestre são fundamentais para as aplicações fotogramétricas. Independentemente do tipo de controle utilizado, um passo interativo é ainda exigido para determinar as coordenadas imagem de pontos de apoio. Esta abordagem propõe uma técnica automática para orientar e ortorretificar imagens terrestres de pontos de apoio. As ortoimagens podem ser utilizadas como cenas de controle em algoritmos de correspondência para orientar imagens aéreas ou orbitais. O método emprega um alvo de controle acoplado a um receptor Global Navigation Satellite System (GNSS) para o levantamento de pontos de apoio. Imagens dos pontos são coletadas em posição nadiral e em diferentes alturas com uma câmara panorâmica. Um modelo multiescala é gerado e orientado usando pontos de enlace e os cantos do alvo como pontos de apoio. As coordenadas 3D destes pontos de enlace são determinadas automaticamente por intersecção de raios. Um Modelo Digital de Terreno (MDT) local é produzido para ortorretificar a área com centro no ponto de apoio. Experimentos foram realizados em pequenas áreas com variações em altitude. As coordenadas geradas automaticamente foram comparadas com amostras coletadas por medições topográficas, e os resultados demonstraram que as áreas foram reconstruídas com erro médio quadrático inferior a $2 \mathrm{~cm}$ em altimetria, o que é compatível com a aplicação proposta de geração de cenas de controle.
\end{abstract}


Palavras-chave: Fotogrametria; Ortoimagem; MDT; Ponto de Apoio; Automação.

\begin{abstract}
Ground control information is essential for photogrammetric applications. No matter the type of control, a manual interactive step is still required to locate image coordinates of Ground Control Points (GCPs). This approach proposes an automatic technique to orientate terrestrial images of GCPs and to generate ortho-images. These ortho-images can be used as control scenes in matching algorithms to orientate aerial and orbital images. The method uses a control target attached to the Global Navigation Satellite System (GNSS) receiver while a GCP is surveyed. GCP images are collected in nadir view and at different heights. A multi-scale model is generated and oriented using tie points and the target as control. The tie point coordinates are automatically determined by intersection of rays. A local Digital Terrain Model (DTM) is produced to orthorectify the area with centre at the GCP. Experiments were performed using small areas with elevation variations. The estimated 3D coordinates were compared to corresponding samples collected by topographic measurements, and the results showed a root mean square errors less than $2 \mathrm{~cm}$ in $\mathrm{Z}$ coordinate for the reconstructed areas, which is compatible with the target applications.
\end{abstract}

Keywords: Photogrammetry; Ortho-Image; DTM; Ground Control Point; Automation.

\title{
1. INTRODUÇÃO
}

A principal fonte de controle geométrico em Fotogrametria tem sido os pontos de apoio. $\mathrm{O}$ controle de campo é essencial nos processos de orientação de imagens e baseia-se na determinação das coordenadas terrestres de elementos fotoidentificáveis.

Dois tipos principais de pontos de apoio são utilizados: pontos pré-sinalizados e pontos naturais. Os pontos pré-sinalizados são materializados no terreno antes da aquisição das imagens aéreas e permitem a automação do processo de identificação e medição, mas têm como desvantagens o alto custo operacional e a restrição de serem instalados antes da realização do voo. Por outro lado, os pontos naturais são elementos bem definidos existentes na área do projeto, mas podem não ter distinguibilidade comparável aos alvos pré-sinalizados e não estar disponíveis nas regiões adequadas do projeto fotogramétrico. Além disso, a automatização de sua localização e medição na imagem não foi resolvida, exceto em sistemas experimentais com exemplos que serão mostrados na seção 2.

Gülch (1995) relatou os principais problemas para a automatização da medição de pontos de apoio. No caso de pontos pré-sinalizados, foram feitas considerações quanto ao modelo, tamanho, forma, fundo, cor e contraste. Já para pontos naturais, estes devem ser únicos e localizáveis com alta precisão. Estão relacionados com feições antrópicas, como marcas de ruas, quinas de construções, interseções de vias. 
Em outros termos, a informação de controle deve ser bem definida em geometria e radiometria, visível em diferentes pontos de vista, ser distinguível e invariante à escala e, ainda, fácil de modelar. Heipke (1997) também fez considerações sobre orientação de imagens para a determinação de Parâmetros de Orientação Exterior (POEs). Algumas importantes condições sobre as fontes de controle foram levantadas e apresentam similaridade com os requisitos previamente apresentados.

Geralmente, os processos manuais interativos ainda são empregados para medir coordenadas imagem de pontos de apoio, o que consome muito tempo, além de proporcionar erros devido à capacidade limitada do operador para interpretar a localização exata dos pontos nas imagens.

Para resolver este problema, propõe-se uma abordagem automática de orientação e geração de cenas terrestres de controle, sem a necessidade de interação de um operador para medir pontos nas imagens. O processo engloba a reconstrução, geração de um Modelo Digital de Terreno (MDT) local, ortorretificação e o georreferenciamento aproximado de imagens terrestres nadirais contendo pontos de apoio.

Com este propósito, uma técnica de coleta de dados foi especialmente desenvolvida para adquirir imagens terrestres nadirais de áreas distinguíveis, usando diferentes alturas para formar um modelo multiescala. Operacionalmente, a técnica, planejada para ser prática e eficiente, não aumenta o tempo de levantamento de campo e utiliza dispositivos de baixo custo.

Objetivamente este artigo introduz um processo automatizado que extrai coordenadas terrestres de alvos, orienta modelos multiescalas e ortorretifica imagens. Uma avaliação também é realizada sobre a reconstrução das coordenadas terrestres em duas pequenas áreas. Como resultado, um elemento de controle terrestre é gerado automaticamente e sem medições manuais. As ortoimagens produzidas podem ser usadas, por exemplo, na orientação de imagens aéreas por meio de algoritmos de correspondência, como apresentado por Berveglieri e Tommaselli $(2013,2014)$.

$\mathrm{Na}$ sequência, relatam-se alguns tipos de elementos de controle usados em Fotogrametria e apresenta-se o método desenvolvido com sua aplicação, resultados e avaliação.

\section{TIPOS DE CONTROLE TERRESTRE}

Na literatura fotogramétrica, diversos tipos de elementos de controle têm sido estudados para os processos de orientação exterior de imagens aéreas ou orbitais. De acordo com Jaw e Wu (2006), além de pontos, outras entidades foram comumente empregadas, como linhas, áreas e estruturas.

Segundo Schenk (2004), a ideia de usar feições como informação de controle em Fotogrametria foi introduzida por Lugnani (1980), o qual demonstrou o uso de feições lineares. Tommaselli e Lugnani (1988) e Tommaselli e Tozzi (1996) utilizaram linhas retas, ao passo que Mulawa e Mikhail (1988) incluíram também as seções cônicas. Outros trabalhos sobre feições lineares também podem ser 
encontrados em Habib e Morgan (2003), Tommaselli e Medeiros (2010) e Marcato Junior e Tommaselli (2013).

Uma das primeiras abordagens utilizando recortes de imagens georreferenciadas foi proposta por Malmström (1986), denominando-os de Ground Control Chip (GCC). O autor utilizou recortes de imagens aéreas de alta resolução para orientar uma imagem de satélite por meio de técnicas de correspondência de imagens baseadas em área.

Drewniok e Rohr (1996) testaram tampas de bueiros como entidades de controle, utilizando um modelo padrão para a correspondência de imagens. Fraser et al. (2001) usaram rotatórias urbanas para avaliar a triangulação de imagens orbitais. Os centroides foram medidos nas imagens e determinados por GPS em campo.

Läbe e Ellenbeck (1996) aplicaram modelos de estruturas 3D de construções como controle para a produção automática de mapas de ortofotos digitais, calculando a orientação exterior por ressecção espacial.

Jaw e Wu (2006) empregaram um banco de recortes de imagens no qual pontos de controle foram localizados em imagens existentes e correlacionados com outras imagens.

Tournaire et al. (2006) propuseram uma abordagem que tem, como controle de campo, faixas de pedestres imageadas por sistemas de mapeamento móvel. A abordagem usou imagens aéreas de alta resolução para gerar um banco de dados de objetos em comum entre imagens aéreas e terrestres.

Li et al. (2013) apresentaram um trabalho sobre o registro de imagens aéreas e dados Light Detection And Ranging (LIDAR) aerotransportados em regiões de deserto. Foram utilizados arbustos existentes no deserto como primitivas de registro. Extraíram-se os centroides desses arbustos para gerar triângulos semelhantes, os quais serviram como características na correspondência entre imagens. A semelhança entre os triângulos foi analisada por lado-ângulo-lado, e a verificação do registro baseou-se no algoritmo RANdom SAmple Consensus (RANSAC).

Essas abordagens previamente apresentadas têm como fatores limitantes, conforme a entidade de controle, a existência da feição de interesse na área do projeto, a disponibilidade de ortoimagens para a área de mapeamento e a dependência pelo nível de resolução e precisão das imagens de referência.

Berveglieri e Tommaselli (2013) apresentaram uma técnica para orientação de imagens aéreas baseada em imagens terrestres de pontos de apoio (de alta resolução), que foram coletadas em campo para extrair recortes georreferenciados para a correspondência com imagens aéreas. Nos experimentos, os pontos de apoio foram localizados automaticamente com precisão subpixel por técnicas de correlação baseada em área e ajustamento por mínimos quadrados. Embora a técnica tenha demonstrado melhorias significativas quando comparada aos métodos tradicionais de medição de pontos, o passo para medir o ponto de apoio nas imagens terrestres ainda era interativo. Para resolver este problema, introduz-se, neste trabalho, uma técnica que gera a cena de controle automaticamente, e a área do

Bol. Ciênc. Geod., sec. Artigos, Curitiba, v. 21, nº 1, p.149-168, jan-mar, 2015. 
entorno do ponto de apoio pode ser usada como controle para orientar imagens aéreas.

\section{MÉTOdO AUTOMÁTICO PARA A ORIENTAÇÃo E GERAÇÃO DE ORTOIMAGENS TERRESTRES DE CONTROLE}

O método proposto utiliza um fluxo completo, desde a orientação à ortorretificação, sem a intervenção de um operador para determinar pontos de apoio nas imagens. Para isso, primeiramente as imagens terrestres devem ser adquiridas com as características específicas da técnica proposta.

A Figura 1 mostra os passos mais importantes do desenvolvimento metodológico. Em termos gerais, quatro etapas foram organizadas para explicar a técnica: (1) processo de calibração da câmara com lente supergrande angular; (2) apoio de campo e forma de aquisição das imagens; (3) orientação das imagens considerando diferentes escalas e, por fim, (4) processo de ortorretificação das regiões dos pontos de apoio.

Figura 1 - Principais passos da metodologia.

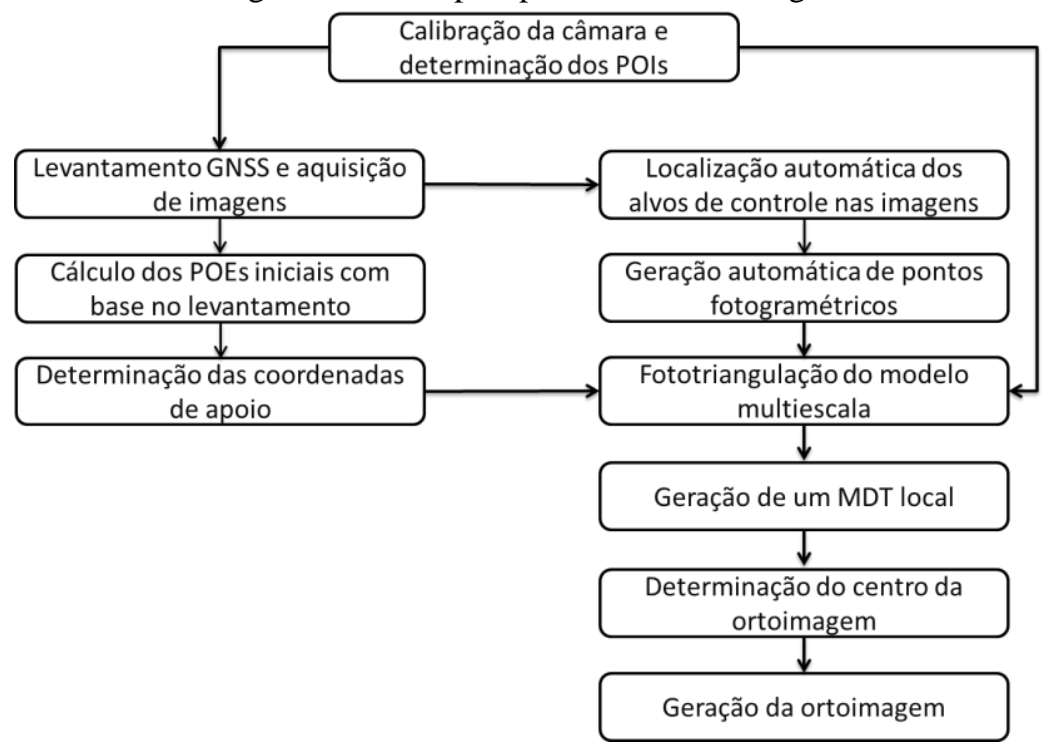

\subsection{Calibração do Sistema Câmara-Lente}

A determinação dos Parâmetros de Orientação Interior (POIs) é feita por um procedimento realizado em campo de calibração terrestre. Em geral, são determinados a distância focal, as coordenadas do ponto principal e os coeficientes de distorção das lentes. O modelo matemático comumente empregado para 
reconstruir a geometria dos raios no interior da câmara baseia-se nas equações de colinearidade (Equação 1) (MIKHAIL et al., 2001), com acréscimo dos parâmetros de distorção das lentes.

$$
\begin{aligned}
& F_{1}=x_{f}-x_{0}-\delta x_{r}-\delta x_{d}-\delta x_{a}+f \frac{m_{11}\left(X-X_{0}\right)+m_{12}\left(Y-Y_{0}\right)+m_{13}\left(Z-Z_{0}\right)}{m_{31}\left(X-X_{0}\right)+m_{32}\left(Y-Y_{0}\right)+m_{33}\left(Z-Z_{0}\right)}=0 \\
& F_{2}=y_{f}-y_{0}-\delta y_{r}-\delta y_{d}-\delta y_{a}+f \frac{m_{21}\left(X-X_{0}\right)+m_{22}\left(Y-Y_{0}\right)+m_{23}\left(Z-Z_{0}\right)}{m_{31}\left(X-X_{0}\right)+m_{32}\left(Y-Y_{0}\right)+m_{33}\left(Z-Z_{0}\right)}=0
\end{aligned}
$$

Em que: $\mathrm{x}_{\mathrm{f}}$ e $\mathrm{y}_{\mathrm{f}}$ são as coordenadas no espaço imagem; (X, Y, Z) são coordenadas do mesmo ponto no espaço objeto; $\mathrm{m}_{\mathrm{ij}}$ são os elementos da matriz de rotação; $\left(\mathrm{X}_{0}, \mathrm{Y}_{0}, \mathrm{Z}_{0}\right)$ são as coordenadas do centro perspectivo da câmara; $\left(\mathrm{x}_{0}, \mathrm{y}_{0}\right)$ são as coordenadas do ponto principal; f é a distância focal e $\mathrm{dx}_{\mathrm{i}}$ e $\mathrm{dy}_{\mathrm{i}}$ representam os efeitos das distorções radial e descentrada e modelo de afinidade.

A lente usada para adquirir imagens é uma supergrande angular do tipo fisheye. Sua geometria tem características particulares que não seguem as condições de colinearidade, exigindo modelagem específica. Para este trabalho, utilizou-se o modelo equidistante (Equação 2) (SCHNEIDER et al., 2009), com adição do modelo de distorção Conrady-Brown (BROWN, 1971).

$$
\begin{aligned}
& x^{\prime}=x_{0}+\delta x_{r}+\delta x_{d}+\delta x_{a}-f \cdot \frac{X_{0}}{\sqrt{X_{0}^{2}+Y_{0}^{2}}} \cdot \arctan \left(\frac{\sqrt{X_{0}^{2}+Y_{0}^{2}}}{Z_{0}}\right) \\
& y^{\prime}=y_{0}+\delta x_{r}+\delta x_{d}+\delta x_{a}-f \cdot \frac{Y_{0}}{\sqrt{X_{0}^{2}+Y_{0}^{2}}} \cdot \arctan \left(\frac{\sqrt{X_{0}^{2}+Y_{0}^{2}}}{Z_{0}}\right)
\end{aligned}
$$

O sistema de equações linearizadas a partir da (Equação 2) pode ser resolvido pelo método dos mínimos quadrados, considerando algumas restrições sobre as coordenadas objeto dos pontos medidos ou observações dos POEs.

A lente supergrande angular é necessária para se obter uma visão ampla, cobrindo as principais características da cena. O processo rigoroso de calibração é fundamental para permitir a correção dos efeitos de distorção radial, próprios da lente fisheye, e do posicionamento da câmara.

\subsection{Levantamento GNSS e Aquisição de Imagens Verticais Multiescalas}

Primeiramente, seleciona-se o local para a coleta do ponto de apoio seguindo os procedimentos convencionais de levantamento: áreas com pontos e feições fotoidentificáveis e abertas (sem obstruções) para evitar problemas na recepção do 
sinal Global Navigation Satellite System (GNSS) e multicaminho. Estas condições favorecem também a aquisição de imagens, evitando problemas com alguma oclusão de objetos.

Após definida a área, uma placa branca $(50 \times 50 \mathrm{~cm})$ contendo um alvo quadrado em preto é colocada e nivelada sobre um tripé e sob a antena do receptor GNSS, como mostram as Figuras 2(a) e (b). Este conjunto de levantamento é posicionado para coletar sinais GNSS sobre uma feição natural bem definida ou sobre uma área que possua em sua vizinhança elementos fotoidentificáveis. Próximo ao receptor, um segundo tripé é posicionado para adquirir imagens com a câmara fisheye em posição nadiral. A Figura 2(c) mostra um exemplo de imagem fisheye adquirida verticalmente com o conjunto montado.

Figura 2 - (a) Dispositivos para aquisição de imagens fisheye em posição vertical e levantamento de ponto de apoio; (b) Placa com o alvo de controle sob a antena GNSS; (c) Um exemplo de imagem vertical fisheye.
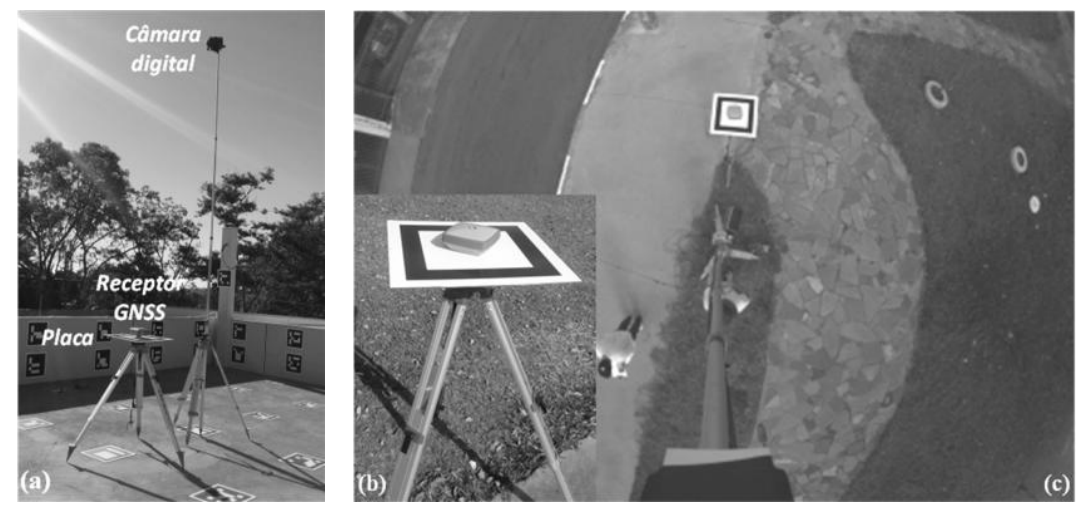

O procedimento de coleta de imagens e sinais GNSS está ilustrado na Figura 3. Depois de posicionada e nivelada, a placa de controle é alinhada perpendicularmente em relação ao tripé da câmara. $\mathrm{O}$ azimute aproximado pode ser medido com uma bússola ou magnetômetro. Enquanto ocorre o rastreio GNSS, quatro imagens em uma posição planimétrica similar são adquiridas, variando-se apenas a altura da câmara. Esta aquisição acontece da seguinte forma:

- Três imagens contendo a placa de controle são tomadas em diferentes alturas, como ilustrado na Figura 3(a);

- Uma quarta imagem, na posição mais alta, é adquirida para ser ortorretificada, sem a presença da placa de controle, rastreador e tripé (ver Figura 3(b));

A definição da necessidade de três alturas foi previamente analisada em campo de calibração, e três imagens foram suficientes para garantir robustez e qualidade geométrica (TOMMASELLI e BERVEGLIERI, 2014). O uso de uma quantidade maior de imagens não produziu melhora significativa. Durante as aquisições, são 
anotadas as medidas de distâncias relativas entre o eixo de centragem do tripé GNSS e o eixo do bastão da câmara (distância D) e as alturas $\left(\mathrm{A}_{\mathrm{I}}\right.$ e $\left.\mathrm{H}_{\mathrm{i}}\right)$.

Figura 3 - (a) Aquisição de três imagens multiescalas registrando a placa de controle. (b) Quarta imagem na posição mais alta e sem a placa de controle. Fonte: Tommaselli e Berveglieri (2014).

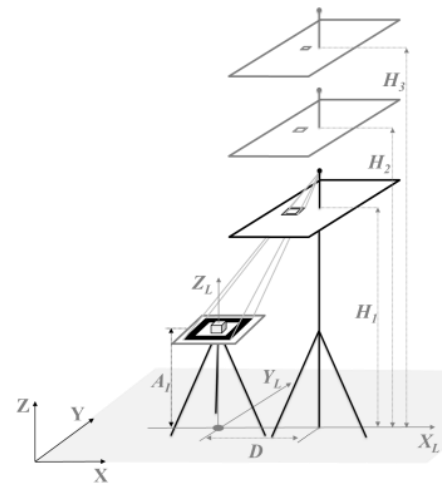

(a)

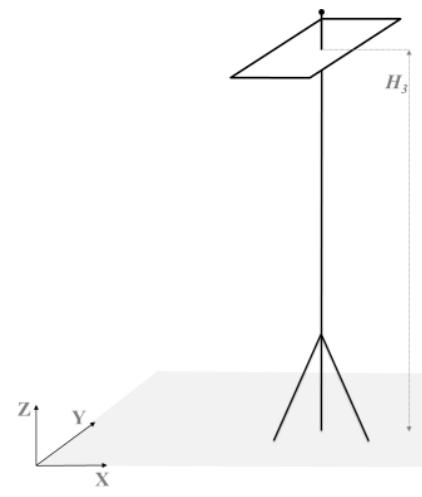

(b)

O posicionamento dos dispositivos e as medições relativas definem um sistema local $\left(\mathrm{X}_{\mathrm{L}}, \mathrm{Y}_{\mathrm{L}}, \mathrm{Z}_{\mathrm{L}}\right)$, que pode ser transformado para outro sistema $(\mathrm{X}, \mathrm{Y}, \mathrm{Z})$, como, por exemplo, para coordenadas geográficas ou coordenadas planas $(E, N, h)$ no sistema Universal Transverse Mercator (UTM). Neste caso, o azimute deve ser corrigido em relação à declinação magnética e à convergência meridiana, ou deve ser calculado com base em uma segunda posição fornecida.

\subsection{Fototriangulação do Modelo Multiescala}

Para realizar a fototriangulação das imagens, são necessários: POIs calibrados, POEs como aproximações iniciais, pontos de apoio e coordenadas imagem como observações.

Os POIs são previamente determinadas pelo processo de calibração da câmara, e os POEs iniciais de posição são medidos diretamente e usados como injunções com base nas medidas anotadas em campo durante a aquisição dos dados. Os POEs de atitude entram na fototriangulação como incógnitas.

Uma vez que o centro da placa tem sua coordenada terrestre definida pelo levantamento GNSS, os oito cantos do alvo quadrado (bordas externa e interna) passam a ter também suas coordenadas conhecidas e são usadas como pontos de apoio para orientar as imagens. Em cada imagem, o alvo de controle é automaticamente identificado por um detector de contornos retangulares, e seus vértices são extraídos (com precisão subpixel) por intersecção de linhas, como mostrado em Garrido-Jurado et al. (2014). 
As quatro imagens são usadas no processo de ajustamento por feixe de raios para estimar os POEs das imagens. A quarta imagem não possui alvos de controle, mas se conecta com as demais pelos pontos de enlace. Um modelo multiescala é então formado, e todas as imagens são conectadas entre si por meio de pontos de enlace, proporcionando mais consistência e rigidez geométrica na estimativa dos POEs. Tais pontos de enlace são determinados automaticamente usando a técnica Scale-Invariant Feature Transform (SIFT).

SIFT é uma técnica de processamento de imagens que extrai automaticamente características distinguíveis baseadas em informações de gradientes locais. Essas características são parcialmente invariantes a mudanças de escala, rotação, iluminação e ponto de vista da câmara. As características extraídas são armazenadas em vetores descritores, e a comparação entre descritores de duas ou mais imagens permite estabelecer a correspondência de imagens. Detalhes sobre a técnica SIFT são apresentados por Lowe (1999, 2004).

É importante ressaltar que o feixe de raios fornecido pela placa de controle nas imagens não tem uma geometria adequada para realizar a orientação completa do modelo. Porém, a orientação é válida para a parte central das imagens, a qual consiste na área do ponto de apoio e abrange as principais características da cena, favorecida pelo posicionamento em campo.

Um estudo para avaliar a acurácia e o alcance desse tipo de orientação foi previamente realizado por Tommaselli e Berveglieri (2014). Demonstrou-se em um campo de calibração, com precisão topográfica de $3 \mathrm{~mm}$, que áreas de até $20 \mathrm{~m}^{2}$ podem ser reconstruídas pela técnica proposta com discrepâncias menores do que $2 \mathrm{~cm}$. Isto é suficiente para o propósito de gerar cenas de controle, já que os levantamentos tradicionais de pontos de apoio têm acurácia inferior a esse valor.

\subsection{Geração da Ortoimagem}

A partir da fototriangulação do modelo multiescala, produtos como MDTs e ortoimagens podem ser gerados. O tamanho do produto depende do nível de precisão a ser atendido, pois a qualidade da orientação tem degradação radial em relação ao centro do alvo de controle. No caso deste estudo, áreas de $20 \mathrm{~m}^{2}$ são suficientes para a correspondência com imagens aéreas de até $20 \mathrm{~cm}$ de Ground Sample Distance (GSD), considerando um levantamento de campo com desviopadrão de $5 \mathrm{~cm}$. Para erros maiores, áreas maiores também podem ser reconstruídas.

\subsubsection{Geração de um MDT Local}

Como já apresentado, os feixes de raios das quatro imagens são ajustados simultaneamente, ligados por pontos de enlace comuns que são determinados por SIFT, os quais podem variar em quantidade conforme as feições da área. $\mathrm{Na}$ fototriangulação do modelo multiescala, todos os pontos de enlace têm suas coordenadas determinadas no espaço objeto por intersecção fotogramétrica, gerando uma nuvem de pontos 3D. 
Originalmente as imagens terrestres são obtidas com GSD entre 2 e $3 \mathrm{~mm}$, de acordo com a altura de aquisição e a distância ao centro da imagem. Esta alta resolução possibilita produzir MDTs locais também com alta resolução, ou gerá-los diretamente para outros GSDs.

Por definição, um MDT deve considerar apenas elementos do terreno. Embora a quarta imagem não contenha o alvo de controle, o tripé e o bastão da câmara ainda estão presentes na imagem. Porém, o posicionamento feito em campo entre os tripés GNSS e câmara favorece que a área de interesse seja sempre visível, sem obstruir as características mais importantes da cena. Assim, a porção da imagem em que tripé e bastão aparecem não produz prejuízos aos propósitos da técnica. Ambos são excluídos usando uma máscara previamente definida, uma vez que esses elementos sempre aparecem na posição centro-inferior da cena. Isto garante que os dispositivos utilizados para coletar dados não contaminem a geração do MDT.

$\mathrm{O}$ processo de produção do MDT segue os procedimentos tradicionalmente conhecidos, como pode ser consultado em Mikhail et al. (2001). Gera-se uma grade regular, conforme as dimensões da área e a resolução pretendida, e aplica-se uma interpolação na grade com base nas coordenadas da nuvem de pontos 3D dos pontos de enlace.

\subsubsection{Geração de uma Ortoimagem Local}

A geração da ortoimagem pode ser feita para uma imagem completa ou para parte dela, como é o interesse deste trabalho, o qual objetiva produzir recortes de imagens terrestres ortorretificadas.

Inicialmente, o procedimento de geração da ortoimagem requer POIs calibrados, o MDT local e a imagem original para ser ortorretificada com seus respectivos POEs.

A finalidade é que seja reconstruída a área circundante ao ponto de apoio. Por isso, a coordenada levantada em campo com receptor GNSS é fornecida para ser localizada no MDT local e determinar o centro de geração da ortoimagem.

De acordo com as dimensões das áreas dos pontos de apoio e da resolução do MDT local, a ortoimagem é produzida. O procedimento adotado usa o método indireto (MIKHAIL et al., 2001) com uma adaptação para gerar a ortoimagem em torno do ponto GNSS levantado. Para cada ponto da ortoimagem no referencial do espaço objeto, faz-se a busca do tom de cinza do pixel correspondente na imagem de entrada seguindo os passos:

1. Projeção das coordenadas $(\mathrm{X}, \mathrm{Y}, \mathrm{Z})$ do MDT local para o espaço imagem usando o modelo equivalente (Equação 2). A coordenada GNSS do ponto de apoio (centro da placa de controle) também é projetada para definir o centro da ortoimagem;

2. Mapeamento inverso conforme o modelo de distorção das lentes para o sistema fotogramétrico distorcido;

3. Determinação da posição no sistema imagem (coluna, linha) com base nas informações da câmara; 
4. Interpolação do tom de cinza a partir dos pixels vizinhos na imagem original.

No final, tem-se a região do ponto de apoio ortorretificada, podendo ser usada como cena de controle.

\section{EXPERIMENTOS E ANÁLISE DE RESULTADOS}

A metodologia proposta foi implementada e testada em situações reais para avaliar todo o processo automático de orientação e geração de ortoimagens em regiões de pontos de apoio.

Observando o espaço abrangido pelas imagens terrestres, se as áreas dos pontos de apoio forem formadas por regiões quase planas, com suaves variações de altitude, um processo de ortorretificação apresenta poucas diferenças sobre a correção dos efeitos do relevo provocados na imagem. Neste caso, a imagem original poderia simplesmente ser reamostrada para um plano, considerando uma mesma escala, o que dispensaria a necessidade de um MDT para ortorretificação. A reamostragem é realizada apenas para corrigir os efeitos resultantes da geometria fisheye e do posicionamento da câmara, gerando uma imagem retificada.

Por outro lado, se algumas variações altimétricas ou, principalmente, inclinações estiverem presentes, então a ortorretificação torna-se necessária para corrigir os efeitos do relevo na imagem. Por isso, considerando este segundo caso, duas áreas com inclinação e algumas variações de altitude na superfície foram selecionadas para serem reconstruídas e avaliadas no espaço objeto. Na sequência, essas áreas serão mostradas, bem como os procedimentos adotados e os resultados alcançados.

\subsection{Realização dos Experimentos}

As imagens para os experimentos foram adquiridas por uma câmara digital com lente fisheye. A Tabela 1 fornece os detalhes técnicos do sistema usado para adquirir imagens.

Tabela 1 - Especificações técnicas do sistema de aquisição de imagens.

\begin{tabular}{l|l}
\hline Modelo da câmara & Nikon D3100 \\
\hline Tamanho do sensor & CMOS APS-C $(23,1 \times 15,4 \mathrm{~mm})$ \\
\hline Dimensões da imagem & $4608 \times 3072$ pixels $(14,2$ megapixels $)$ \\
\hline Tamanho do pixel & $0,005 \mathrm{~mm}$ \\
\hline Distância focal nominal & $8,0 \mathrm{~mm}$ (Bower SLY 358N) \\
\hline
\end{tabular}

A calibração da lente fisheye foi realizada em um campo de calibração terrestre 3D, localizado na FCT/UNESP em Presidente Prudente/SP. O campo possui 139 alvos com codificação ARUCO (GARRIDO-JURADO et al., 2014), como mostra a Figura 4. Este tipo codificação possibilita identificar os alvos e medir as coordenadas dos cantos para serem usadas como controle no ajuste de feixe de raios na calibração do sistema câmara-lente. 
Um programa computacional de localização de alvos ARUCO, usando biblioteca OpenCV, foi adaptado por Silva et al. (2014) para calibração de câmaras. Os quatro cantos de cada alvo foram automaticamente localizados e medidos nas imagens, fornecendo suas respectivas coordenadas terrestres. Do mesmo modo, o alvo da placa de controle também foi planejado para ser identificado automaticamente.

Figura 4 - Exemplo de uma imagem fisheye com alvos ARUCO usada para calibração da câmara.

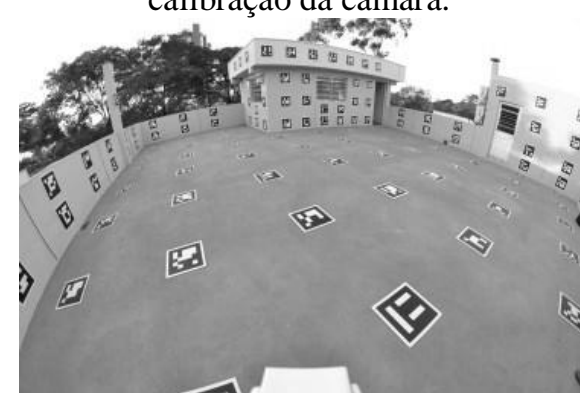

A partir das coordenadas identificadas, os POIs foram determinados usando o software Calibration with Multiple Cameras (CMC), desenvolvido por Ruy et al. (2009), com adaptação feita por Tommaselli et al. (2014) para o modelo equidistante, apropriado para a lente fisheye. Os valores dos POIs estimados pelo processo de calibração estão apresentados na Tabela 2. Para mais detalhes sobre essa calibração, ver Tommaselli e Berveglieri (2014).

Tabela 2 - POIs estimados pelo processo de calibração usando ajuste de raios com o modelo equidistante.

\begin{tabular}{l|l|l}
\hline Parâmetro & Valor estimado & Desvio-padrão estimado \\
\hline $\mathrm{f}(\mathrm{mm})$ & 8,3794 & $0,0011( \pm 0,23$ pixels $)$ \\
\hline $\mathrm{x}_{0}(\mathrm{~mm})$ & 0,0729 & $0,0011( \pm 0,22$ pixels $)$ \\
\hline $\mathrm{y}_{0}(\mathrm{~mm})$ & 0,0019 & $0,0009( \pm 0,18$ pixels $)$ \\
\hline $\mathrm{K}_{1}\left(\mathrm{~mm}^{-2}\right)$ & $4,20 \times 10^{-4}$ & $3,77 \times 10^{-6}$ \\
\hline $\mathrm{K}_{2}\left(\mathrm{~mm}^{-4}\right)$ & $8,20 \times 10^{-7}$ & $6,32 \times 10^{-8}$ \\
\hline $\mathrm{K}_{3}\left(\mathrm{~mm}^{-6}\right)$ & $-2,54 \times 10^{-9}$ & $3,12 \times 10^{-10}$ \\
\hline $\mathrm{P}_{1}\left(\mathrm{~mm}^{-1}\right)$ & $4,84 \times 10^{-6}$ & $1,63 \times 10^{-6}$ \\
\hline $\mathrm{P}_{2}\left(\mathrm{~mm}^{-1}\right)$ & $-2,28 \times 10^{-7}$ & $1,67 \times 10^{-6}$ \\
\hline $\mathrm{A}$ & $3,34 \times 10^{-5}$ & $1,88 \times 10^{-5}$ \\
\hline $\mathrm{B}$ & $-7,43 \times 10^{-4}$ & $3,58 \times 10^{-5}$ \\
\hline$\sigma$ a posteriori & $0,0054(\approx 1$ pixel $)$ & \\
\hline
\end{tabular}


$\mathrm{Na}$ aquisição das imagens das duas áreas de estudo, as alturas da câmara variaram em torno de: $3,1 \mathrm{~m} ; 3,7 \mathrm{~m}$ e 4,4 $\mathrm{m}$ (alturas medidas com distanciômetro eletrônico), ressaltando-se que a terceira e quarta imagens são adquiridas na mesma altura. A definição destes valores aproximados teve como base os experimentos realizados por Tommaselli e Berveglieri (2014) no campo de calibração. Para o levantamento das coordenadas dos pontos de apoio, um receptor GNSS (Hiper SR Topcon) de dupla-frequência foi usado.

O conjunto de imagens de cada ponto de apoio forma um modelo multiescala. Assim, a técnica SIFT foi aplicada sobre cada modelo para gerar pontos de enlace automaticamente, e os resultados da correspondência aparecem nas Figuras 5(a) e (b) para ambas as áreas, considerando as imagens de controle, as quais também tiveram os cantos da placa de controle localizados por processamento de imagens. $\mathrm{Na}$ correspondência SIFT, possíveis falsas correspondências foram eliminadas usando uma filtragem combinada com transformação de Helmert e majority voting, implementada por Berveglieri e Tommaselli (2014).

Figura 5 - Pontos de enlace entre as três imagens do modelo com a placa de controle. São mostradas as regiões: (a) Área I; (b) Área II; (c) e (d) Respectivas áreas ortorretificadas com GSD de $1 \mathrm{~cm}$ e centro no ponto de apoio.

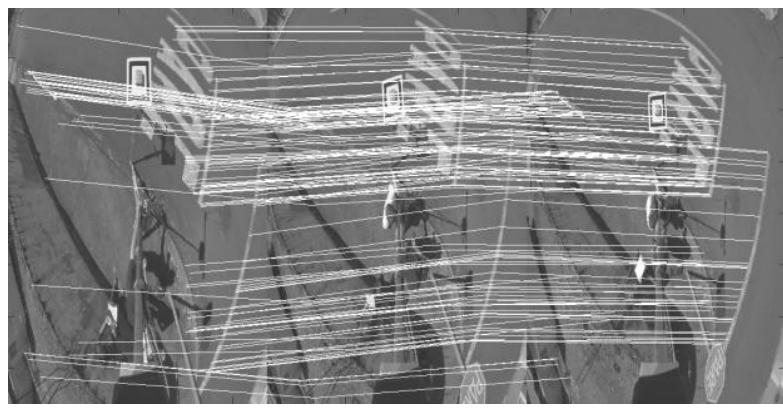

(a)

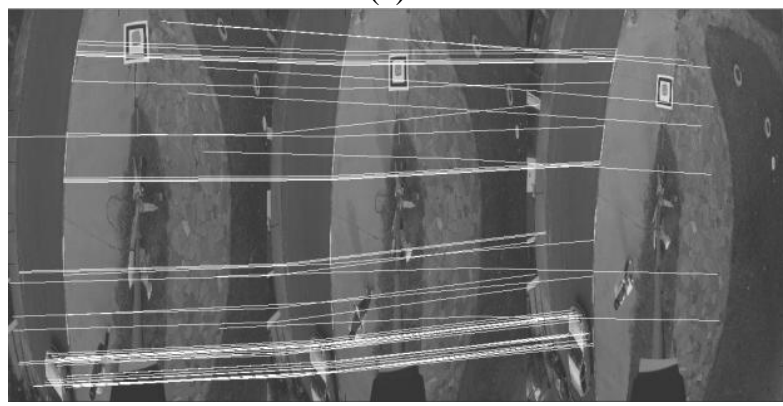

(b)

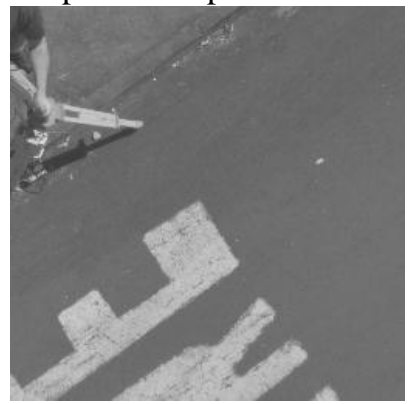

(c)

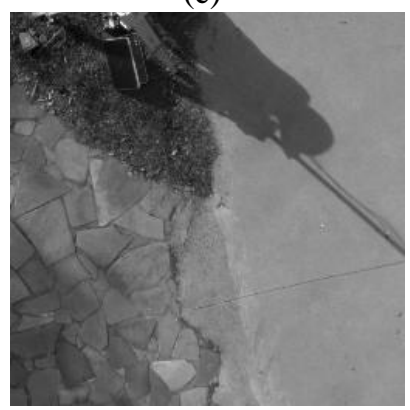

(d) 
As coordenadas imagem de cada imagem foram inseridas no projeto fotogramétrico como observações para processar a triangulação pelo programa CMC. Nesse procedimento de fototriangulação, definiu-se, no espaço imagem, o desvio-padrão de $\sigma=0,5$ pixel para as coordenadas imagem. Em relação ao espaço objeto, as coordenadas de apoio sobre a placa de controle (oito cantos), por serem conhecidas com precisão, foram fixadas por injunções com desvio-padrão de $\sigma=0,0005 \mathrm{~m}$. Os valores iniciais dos POEs foram definidos com base na determinação direta das medidas obtidas em campo, sendo fixados por injunções relativas com desvios-padrão de: $\sigma=30^{\circ}$ para a atitude, que não afeta significativamente os resultados; e devido aos movimentos e deslocamentos para levantar a câmara, $\sigma=0,10 \mathrm{~m}$ para a posição XY e $\sigma=0,005 \mathrm{~m}$ para Z. Usando fotriangulação por feixes de raios, cada modelo multiescala foi ajustado, resultando nos sigmas a posteriori de $\hat{\sigma}_{0}=0,0096\left(<2\right.$ pixels) e $\hat{\sigma}_{0}=0,0137(<3$ pixels $)$ para as Áreas I e II, respectivamente, sendo que o sigma a priori atribuído foi de $\sigma_{0}=0,0142(\sim 3$ pixels), devido aos efeitos de borramento da lente fisheye.

As coordenadas terrestres dos pontos de enlace da região do ponto de apoio foram determinadas por intersecção fotogramétrica no espaço objeto, gerando uma nuvem de pontos. Para cada área, a partir das nuvens geradas, interpolou-se um MDT local em forma de grade regular a com elemento de resolução de grade regular de $1 \mathrm{~cm}$. A técnica de interpolação usada foi a ponderação pelo inverso do quadrado da distância. Outro espaçamento também poderia ser utilizado, uma vez que as imagens originais possuem GSD entre 2 e $3 \mathrm{~mm}$. Entretanto, definiu-se a resolução em $1 \mathrm{~cm}$ apenas por uma questão prática, permitindo facilmente a subamostragem da imagem para qualquer outro GSD.

Desse modo, utilizando-se o procedimento descrito na seção 3.4, a ortoimagem foi produzida segundo as dimensões da área de interesse. As Figuras 5(c) e (d) apresentam as ortoimagens geradas com o ponto de apoio no centro.

É recomendado produzir uma ortoimagem com alta resolução (por exemplo, GSD de $1 \mathrm{~cm}$ ) para depois subamostrá-la para outras resoluções (GSD de $3 \mathrm{~cm}$, $4 \mathrm{~cm}$ ou maior). Experimentalmente, verificou-se que a geração de uma ortoimagem diretamente na resolução pretendida (GSD de $3 \mathrm{~cm}, 4 \mathrm{~cm}$ ou maior) resultou em uma qualidade inferior em comparação com a mesma imagem produzida com elemento de resolução de $1 \mathrm{~cm}$ e, posteriormente, subamostrada para aqueles GSDs.

\subsection{Validação das Ortoimagens e Análise dos Resultados}

Para validar as ortoimagens locais, conduziu-se um levantamento topográfico por nivelamento trigonométrico para se obter amostras discretas das altitudes das áreas experimentais. A escolha do método adotado foi devido às pequenas dimensões das áreas.

Em campo, posicionou-se no mesmo ponto de levantamento GNSS uma estação total (Topcon GPT-7005) para fazer o levantamento por irradiação de pontos no entorno, os quais eram ocupados por um bastão com prisma refletor. A 
região do ponto de apoio (área com cerca de $5 \times 5 \mathrm{~m}$ ) foi levantada, coletando-se coordenadas de pontos com espaçamento de 0,5 ou $1 \mathrm{~m}$, no máximo. A Figura 6 exibe a variação da superfície das duas áreas de teste, a partir do levantamento de campo.

Como já mencionado, devido ao tipo de geometria da placa de controle, que é posicionada para a parte central das imagens, apenas o entorno dos pontos de apoio é reconstruído. Não é finalidade reconstruir todo o espaço coberto pelo modelo multiescala, pois as áreas de interesse apresentam dimensões de $2 \times 2 \mathrm{~m}$ ou $3 \times 3 \mathrm{~m}$ e são recortadas e usadas como templates ou chips de imagens.

Figura 6 - MDTs das áreas de teste com tamanho aproximado de $5 \times 5 \mathrm{~m}$. (a) Área I e (b) Área II.

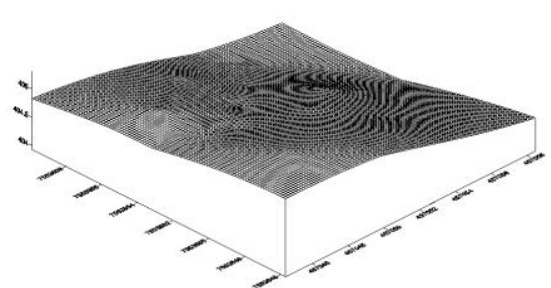

(a)

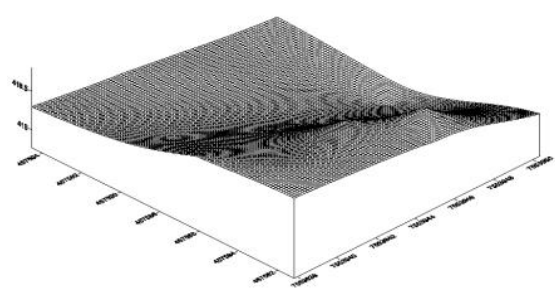

(b)

Pelo fato dos pontos de enlace serem determinados automaticamente e não serem necessariamente cantos, sua localização exata em campo não é possível. Desse modo, as coordenadas topograficamente medidas foram interpoladas para as mesmas posições das coordenadas 3D geradas pela triangulação do modelo. As diferenças entre as coordenadas trianguladas e as interpoladas possibilitaram uma avaliação sobre a qualidade do MDT local e da ortoimagem.

As amostras de campo foram interpoladas espacialmente usando a média ponderada das quatro amostras mais próximas ao ponto em análise. Os pesos foram definidos baseados no inverso do quadrado da distância, e um cálculo de discrepâncias foi realizado considerando as coordenadas topográficas interpoladas como referência. A partir disso, calculou-se a Raiz do Erro Médio Quadrático (REMQ) das altitudes dos pontos.

Os resultados estatísticos da avaliação estão apresentados na Tabela 3. O número de pontos de enlace foi definido pelo processo de geração automática, que depende das feições distinguíveis de cada área. As discrepâncias das estimativas altimétricas na região ortorretificada indicaram REMQ inferiores a $2 \mathrm{~cm}$ para as Áreas I e II. A maior média nas discrepâncias foi obtida na Área I, sendo igual a $6 \mathrm{~mm}$, e os desvios-padrão de ambas as áreas resultaram em valores também abaixo de $2 \mathrm{~cm}$. 
Tabela 3 - Resultado das discrepâncias altimétricas calculadas pela diferença entre as coordenadas trianguladas e as interpoladas topograficamente.

\begin{tabular}{c|c|c|c|c}
\hline Ortoimagem & $\mathrm{N}^{\mathbf{o}}$ de pontos & REMQ $(\mathrm{m})$ & Média $(\mathrm{m})$ & Desvio-padrão $(\mathrm{m})$ \\
\hline Área I & 14 & 0,015 & 0,006 & 0,014 \\
\hline Área II & 8 & 0,019 & 0,002 & 0,019 \\
\hline
\end{tabular}

Na Figura 7, são apresentados os mapas de discrepâncias entre os modelos de terreno levantados em campo e os modelos determinados a partir dos pontos de enlace, que também são mostrados nestes gráficos. Na comparação direta com as amostras, a Área I apresentou discrepâncias entre -1,2 e 3,4 cm, enquanto a Área II apresentou valores variando no intervalo de $-3,7$ a $2,7 \mathrm{~cm}$.

Figura 7 - Mapa das discrepâncias em Z (em metros): (a) Área I e (b) Área II, mostrando pontos de enlace gerados automaticamente.

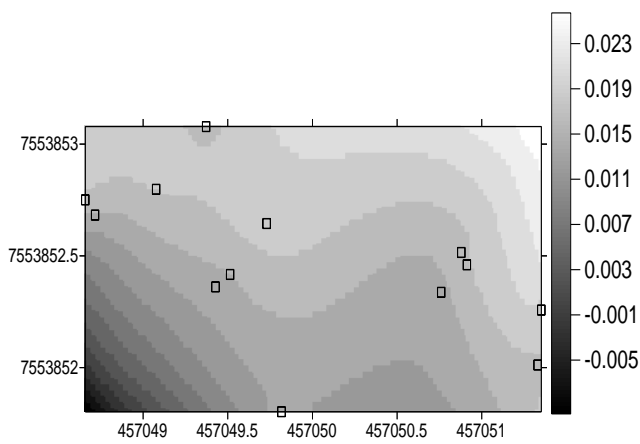

(a)

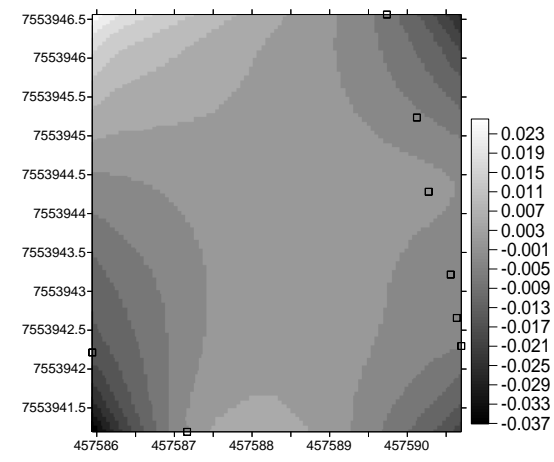

(b)

A partir dos MDT gerados, das imagens digitais e respectivos parâmetros (POE e POI), as ortoimagens foram produzidas com as orientações próximas ao norte geográfico. É importante ressaltar que o pixel central da ortoimagem tem coordenadas precisas, pois corresponde ao ponto de controle levantado com GNSS, mas os demais pixels podem apresentar uma pequena rotação, devido às incertezas nas medições do azimute aproximado no levantamento de campo. Esta rotação não afetará o processo posterior de correspondência com imagens aéreas ou orbitais, porque os algoritmos de matching por mínimos quadrados (Least Squares Matching - LSM) absorverão esta pequena rotação, como mostrado por Berveglieri e Tommaselli (2013). Entretanto, esse problema pode ser facilmente resolvido pela introdução de uma segunda antena GNSS junto à câmara. O posicionamento relativo entre a antena GNSS da câmara e a antena GNSS sobre a placa de controle possibilita obter um azimute acurado da imagem.

Em seguida, recortes ao redor do ponto de controle podem ser feitos para extrair templates. Experimentos com a correspondência multiescala entre imagens 
terrestres e aéreas foram desenvolvidos por Berveglieri e Tommaselli (2013). Nesse tipo de aplicação, foi demonstrado que pontos de apoio podem ser automaticamente localizados com precisão subpixel por métodos de correspondência e alguns refinamentos. A técnica apresentada, neste artigo, conecta-se a esta previamente citada, pela fase anterior ao processo de localização de pontos, a qual consiste em localizar e medir automaticamente o ponto de apoio na imagem sem usar, portanto, técnicas interativas de medição. Dessa forma, erros manuais de medição são eliminados, e áreas distinguíveis podem ser usadas como elemento de controle terrestre para orientar imagens.

\section{CONCLUSÕES}

Uma técnica automática para a fototriangulação de imagens terrestres verticais e geração de ortoimagens de pontos de apoio foi apresentada e avaliada.

Considerando as dimensões das imagens que são adquiridas pela câmara fisheye, em alguns cenários de pontos de apoio, pequenas irregularidades da superfície podem não afetar significativamente as imagens pelo deslocamento radial devido ao relevo. Entretanto, quando há variações altimétricas significativas, a escala da imagem tem diferentes valores, conforme a distância radial em relação ao centro da imagem. Neste caso, a ortorretificação é necessária e foi avaliada em situações reais.

Experimentalmente, a técnica demonstrou em campo praticidade para a montagem e coleta de imagens e, ainda, tempo compatível com o levantamento GNSS. A aquisição de imagens com deslocamentos verticais evitou a necessidade de translação do sistema para aquisição de estéreo-pares, e verificou-se que nenhum operador especializado seria exigido para manipular o sistema, podendo ser o próprio topógrafo.

Uma placa de controle foi introduzida como parte do método. Embora a geometria provida pelos alvos não seja ótima, é aceitável para reconstruir a área do entorno do ponto de apoio. As análises estatísticas indicaram REMQ inferior a $2 \mathrm{~cm}$ nas discrepâncias altimétricas entre o MDT gerado e os dados de campo. O tamanho proporcionado também é suficiente para extrair recortes de imagens (ou templates) para servirem como cenas de controle.

Nesta técnica, apenas áreas com características distinguíveis precisam ser selecionadas. A placa de controle pode ser posicionada em qualquer local próximo às principais feições da área. Como resultado, um banco de ortoimagens de pontos de apoio com altíssima resolução (GSD $3 \mathrm{~mm}$ ) pode ser produzido.

Nos procedimentos apresentados, desenvolveu-se um fluxo automático, partindo da localização dos pontos de apoio até a ortorretificação de imagens, sem interações manuais para medir coordenadas, eliminando o erro proporcionado por um operador nessa tarefa. Em trabalhos futuros, todo esse fluxo automático será testado na orientação de blocos de imagens para avaliar sua acurácia global.

\section{AGRADECIMENTOS}

Bol. Ciênc. Geod., sec. Artigos, Curitiba, v. 21, nº 1, p.149-168, jan-mar, 2015. 
Os autores agradecem ao Conselho Nacional de Desenvolvimento Científico e Tecnológico $(\mathrm{CNPq}$ - processo 305111/2010-8) e à Fundação de Amparo à Pesquisa do Estado de São Paulo (FAPESP - processo 2010/16954-5) pelo apoio financeiro na forma de bolsa de estudos.

\section{REFERÊNCIAS BIBLIOGRÁFICAS}

BERVEGLIERI, A.; TOMMASELLI, A. M. G. Multi-scale matching for the automatic location of control points in large scale aerial images using terrestrial scenes. In: International Archives of Photogrammetry, Remote Sensing and Spatial Information Sciences. v. XL-3/W1. p. 27-31, 2014.

BERVEGLIERI, A.; TOMMASELLI, A. M. G. Automatic measurement of ground control points with terrestrial control chips for bundle block triangulation. In: Joint CIG Annual Conference and EOGC'2013. Proceedings..., Toronto, 2013.

BROWN, D. C. Close-range calibration. Photogrammetric Engineering, v. 37, n. 8, p. 855-866, 1971.

DREWNIOK, C.; ROHR, K. Automatic Exterior Orientation Of Aerial Images In: International Archives of Photogrammetry and Remote Sensing, v. 31(B3), Viena, p.146-152, 1996.

FRASER, C. S.; HANLEY, H. B.; YAMAKAWA, T. Sub-metre geopositioning with ikonos geo imagery. ISPRS Joint Workshop, 2001. Proceedings..., Hannover, 2001.

GARRIDO-JURADO, S.; MUÑOZ-SALINAS, R.; MADRID-CUEVAS, F. J.; MARÍN-JIMÉNEZ, M. J. Automatic generation and detection of highly reliable fiducial markers under occlusion. Pattern Recognition. v. 47, p. 2280-2292, 2014.

GÜLCH, E. Automatic Control Point Measurement. In: Photogrammetric Week'95. Heidelberg, p. 185-196, 1995.

HABIB, A. F.; MORGAN, M. F. Linear features in photogrammetry. Boletim de Ciências Geodésicas, v. 9, n. 1, p. 3-24, 2003.

HEIPKE, C. Automation of interior, relative, and absolute orientation. ISPRS Journal of Photogrammetry and Remote Sensing, v. 52, n. 1, p. 1-19, 1997.

JAW, J.-J.; WU, Y.-S. Control pacthes for automatic single photo orientation. Photogrammetric Engineering \& Remote Sensing, v. 72, n. 2, p. 151-157, 2006.

LÄBE, T.; ELLENBECK, K. H. 3D-wireframe models as ground control points for the automatic exterior orientation. In: International Archives of Photogrammetry and Remote Sensing. v. 31(B2), Vienna, p.218-223, 1996.

LI, N.; HUANG, X.; ZHANG, F.; WANG, L. Registration of aerial imagery and lidar data in desert areas using the centroids of bushes as control information. Photogrammetric Engineering \& Remote Sensing, v. 79, n. 8, p. 743-752, 2013.

LOWE, D. G. Object Recognition from Local Scale-Invariant Features. In: Seventh IEEE International Conference on Computer Vision, v.2, p. 1150-1157, 1999

LOWE, D. G. Distinctive Image Features from Scale-Invariant Keypoints. Int. J. Comput. Vision, v. 60, n. 2, p. 91-110, 2004. 
LUGNANI, J. B. Using digital entities as control, 1980. PhD Thesis, Fredericton: University of New Brunswick, Canada.

MALMSTRÖM, H. Measuring ground control points for satellite image rectification. Schriftenreihe des Instituts für Photogrametrie der Universität Stuttgart. Proceedings... v. 11, p.127-135, 1986.

MARCATO JUNIOR, J.; TOMMASELLI, A. M. G. Exterior orientation of CBERS-2B imagery using multi-feature control and orbital data. ISPRS Journal of Photogrammetry and Remote Sensing, v. 79, p. 219-225, 2013.

MIKHAIL, E. M.; BETHEL, J. S.; MCGLONE, C. J. Introduction to modern photogrammetry. New York: John Wiley \& Sons, 2001, 479p.

MULAWA, D. C.; MIKHAIL, E. M. Photogrammetric treatment of linear features. In: International Archives of Photogrammetry, Remote Sensing and Spatial Information Sciences. v. 27 (Part B3), p. 383-393, 1988.

SCHENK, T. From point-based to feature-based aerial triangulation. ISPRS Journal of Photogrammetry and Remote Sensing, v. 58, p. 315-329, 2004.

SCHNEIDER, D.; SCHWALBE, E.; MAAS, H. G. Validation of geometric models for fisheye lenses. ISPRS Journal of Photogrammetry and Remote Sensing, v. 64, n. 3, p. 259-266, 2009.

SILVA, S. L. A.; TOMMASELLI, A. M. G.; ARTERO, A. O. Utilização de alvos codificados na automação do processo de calibração de câmaras. Boletim de Ciências Geodésicas, v. 20, n. 3, p. 636-656, 2014.

RUY, R. S.; TOMMASELlI, A. M. G; GALO, M.; HASEGAWA, J. K.; REIS, T. T. Evaluation of bundle block adjustment with additional parameters using images acquired by SAAPI system. In: 6th International Symposium on Mobile Mapping Technology, 2009, Presidente Prudente. Proceedings..., Presidente Prudente, 2009.

TOMMASELLI, A. M. G.; MARCATO JUNIOR, J.; MORAES, M. A. V.; SILVA, S. L. A.; ARTERO, A. O. Calibration of panoramic cameras with coded targets and a 3D calibration field. In: International Archives of Photogrammetry, Remote Sensing and Spatial Information Sciences. v. XL-3/W1. p. 137-142, 2014.

TOMMASELLI, A. M. G.; MEDEIROS, N. G. Determination of the indirect orientation of orbital pushbroom images using control straight lines. The Photogrammetric Record, v. 25, n. 130, p. 159-179, 2010.

TOMMASELLI, A. M. G.; BERVEGLIERI, A. Automatic orientation of multi-scale terrestrial images for 3D reconstruction. Remote Sensing, v. 6, n. 4, p. 3020-3040, 2014.

TOMMASELLI, A. M. G.; LUGNANI, J. An alternative mathematical model to collinearity equations using straight features. In: International Archives of Photogrammetry and Remote Sensing, 1988, Part B3, p.765-774, 1988.

TOMMASELLI, A. M. G.; TOZZI, C. L. A recursive approach to space resection using straight lines. Photogrammetric Engineering \& Remote Sensing, v. 62, n.1, p. 57-66, 1996. 
TOURNAIRE, O.; SOHEILLIAN, B.; PAPARODITIS, N. Towards a subdecimetric georeferencing of ground-based mobile mapping systems in urban areas: matching ground-based and aerial-based imagery using roadmarks. In: International Archives of Photogrammetry and Remote Sensing. Proceedings..., Paris, 2006.

(Recebido em março de 2014. Aceito em dezembro de 2014). 